\title{
Ockham, Suárez y Descartes: transición de las noéticas tardomedievales a la filosofía moderna
}

\author{
Ockham, Suarez and Descartes: the transition \\ from the late medieval Noethics to modern \\ Philosophy
}

Leopoldo José Prieto López

Universidad Francisco de Vitoria

Recibido: 09-02-2015

Aceptado: 26-09-2015

\section{Resumen}

El artículo estudia algunas ideas de importancia de las teorías del conocimiento del siglo XIV que se estima tienen un papel relevante en el origen de la filosofía moderna, particularmente en la filosofía de Descartes. Tales ideas, entre otras, son: a) la noción escotista de notitia abstractiva y su correlato en la noción de conceptus objectivus de procedencia aviceniana; b) la tendencia presente en un buen número de autores franciscanos, que en el proceso del conocimiento otorga más relevancia al sujeto cognoscente que a la cosa conocida; c) la idea teológica de la omnipotencia divina que, de potentia absoluta, aplicada al conocimiento humano, cree posible un concepto sin objeto; d) una difusa tendencia al escepticismo, que se alimenta de dos fuentes: la teoría agustiniana de la iluminación y el giro que en el siglo XIV experimenta la lógica hacia la dialéctica. En torno a estas ideas se descubre una línea de pensamiento común a Ockham, Suárez (y los conimbricenses) y Descartes, en cuyas filosofías la intencionalidad y la certeza del conocimiento quedan comprometidas.

Palabras clave: Avicena, Escoto, Olivi, Aureolus, Ockham, Suárez, Descartes, conceptus objectivus, esse objectivum, intencionalidad, certeza. 


\begin{abstract}
The article studies some important ideas of the theory of knowledge in the fourteenth century that are estimated to be particularly relevant in the origin of modern philosophy, especially in the philosophy of Descartes. Among others, some of the ideas are: a) the Scotus notion of notitia abstractiva and its correlation with the avicennan conceptus objectivus; b) the trend present in a substantial amount of Franciscan authors, that, in the process of knowledge, puts the emphasis more in the knower than thing known; c) the theological idea of divine omnipotence that, de potentia absoluta, applied in human knowledge, believes that it is possible to know without an object; d) a diffuse tendency to the scepticism, which is based on two sources: the Augustinian theory of the illumination and the change that in the fourteenth century the logic experiences moving forward to the dialectic. In these ideas we discover a common line among Ockham, Suárez (and the conimbricenses) and Descartes, in whose philosophies the intentionality and the certitude of knowledge are jeopardised.
\end{abstract}

Keywords: Avicenna, Scotus, Olivi, Aureolus, Ockham, Suárez, Descartes, conceptus objectivus, esse objectivum, intentionality, certitude.

\title{
1. Planteamiento general
}

La filosofía moderna, inspirada profundamente por el principio de inmanencia del conocimiento, es deudora de la filosofía del siglo XIV, en la que este principio comienza a tomar cuerpo. En este trabajo pretendo dar a conocer algunas ideas del pensamiento tardomedieval que, según creo, se encuentran en el origen de la filosofía moderna.

Dos son los rasgos que, en mi opinión, constituyen las coordenadas esenciales del pensamiento moderno: una teoría del conocimiento anclada en el principio de inmanencia y una teoría de la naturaleza que privilegia una lectura meramente matemática de la misma, que es además la clave profunda de la venidera revolución científica del siglo XVII.

Pues bien, de la primera coordenada, que es la que aquí nos interesa, podemos decir que empieza a fraguarse en el siglo XIV, a partir de algunas ideas tardomedievales como son las siguientes.

a) La teoría de Escoto sobre la notitia abstractiva introduce una noción de conocimiento que empieza a perder contacto con las realidades naturales, precisamente porque la abstracción es entendida por Escoto no tanto como un procedimiento de universalización a partir de las realidades naturales, sino como un conocimiento de 
las cosas que, obtenido en ausencia de las mismas, es mediado por una repraesentatio cuya relación con la realidad material es cada vez más débil.

b) Una tendencia general de origen agustiniano en la teoría del conocimiento, característica de la escuela franciscana, en virtud de la cual se entiende que en el proceso del conocimiento es de mayor relevancia la aportación del sujeto cognoscente que la de la cosa conocida. En este sentido Enrique de Gante entiende que el acto intelectivo parte del alma y se consuma en ella. En tal sentido la cosa conocida es más ocasión que causa de conocimiento. La prioridad en el acto del conocimiento se otorga al sujeto1. De un modo más matizado, pero en esta misma dirección, entiende Escoto que, siendo el entendimiento causa simpliciter más perfecta que la cosa, la eficacia del entendimiento resulta de mayor incidencia en el acto de conocimiento que la de la cosa ${ }^{2}$. Escoto llega incluso a comprometer la intervención de la cosa cuando afirma que ésta es una suerte de instrumento del mismo entendimiento.

Pedro Aureolo, a su vez, elabora una noética cuyo propósito fundamental es reducir tanto como sea posible la eficacia de la cosa en la producción del concepto, para dar así más relevancia sea a la actividad de la mens (el apex intellectus, que, en un sentido agustiniano, no se relaciona con las cosas, sino que se ocupa de sí mismo) sea a la omnipotencia de Dios, que puede dispensar al acto de conocimiento del contacto inmediato con la cosa (a lo que Aureolo, inspirándose claramente en Escoto, llama la existencia y presencia de la cosa). El gusto agustiniano del razonamiento de Aureolo es evidente, como agustiniano será el estilo de la reflexión cartesiana sobre la actividad del cogito, que, aislado del mundo, descubre dentro de sí unos cogitata, algunos de los cuales podrían haber sido producidos por la voluntad de un ser sumamente poderoso y astuto que nos hiciera concebir una determinada idea sin un objeto exterior con el que guardara correspondencia alguna. Las doctrinas cartesianas de la realitas objectiva de la idea y del mauvais génie hunden aquí sus raíces.

Por su parte, la teoría del conocimiento de Olivi, más aún que la de Aureolo, disminuye al mínimo, o incluso anula, la actividad causal del objeto sobre el espíritu, poniendo del lado de éste la eficiencia en el acto de conocimiento. R. Pasnau y J. Tovainen afirman inequívocamente de la noética de Olivi que niega toda cau-

\footnotetext{
1 En tal sentido, innatismo (también el virtual de Leibniz y del mismo Kant) y ocasionalismo encuentran asiento posible en esta caracterización del conocimiento y se encuentran prefigurados in nuce en esta doctrina.

2 Cf. Escoto, Reportata Parisiensia, sumptibus Laurentii Durand, Ludguni, 1639, lib. I, dist. III, q. 1, sch. 2, 43: "Impossibile est quodcumque obiectum facere in intellectu nostro conceptum perfectiorem suo proprio conceptu quiditativo, quia causa aequivoca naturaliter agens non potest effectum producere perfectiorem suo proprio effectu sibi similissimo, et sibi in virtute sua adaequato. Igitur, cum obiectum sit causa aequivoca respectu sui conceptus proprii quiditativi, non potest conceptum perfectiorem in intellectu nostro alterius obiecti gignere".
} 
salidad de la cosa sobre el espíritu. "El objeto no ejerce influjo causal alguno sobre la facultad de conocimiento ni sobre los órganos físicos del sentido. El objeto externo no requiere más que estar lo suficientemente próximo para ser aprehendido por la atención espiritual del cognoscente. Tanto en el caso de la sensación como de la intelección, el agente eficiente es la facultad cognoscitiva"3.

c) Por otro lado, apelando a la omnipotencia divina, según el tipo de razonamiento de potentia Dei absoluta que se hace común por influjo del voluntarismo en general y de Guillermo de Ockham en particular, comienza a admitirse en el siglo XIV que es posible el conocimiento de un objeto no ya en ausencia del mismo, como había sostenido Escoto, sino no existiendo objeto alguno que cause la representación. La posibilidad de un concepto sin objeto, es decir, de un concepto al que no corresponde realidad alguna exterior al espíritu, es una revolución teórica de magnitud formidable que, inspirada inicialmente en motivos teológicos, se prolongará a lo largo de la edad moderna en la historia del pensamiento.

d) Finalmente, una generalizada tendencia al escepticismo, que caracteriza a todo el siglo XIV, refuerza el movimiento de reversión del espíritu sobre sí mismo en la búsqueda de la verdad implicada en las ideas anteriores. Del escepticismo del siglo XIV hay que decir que se alimenta principalmente de dos fuentes: de un lado, de la teoría del conocimiento de san Agustín; y de otro, de ciertas ideas lógicas que critican y ponen en duda el valor de las demostraciones hasta entonces dadas en metafísica.

Michalski ha llamado la atención sobre el hecho de que la teoría del conocimiento de san Agustín encierra los gérmenes de aquel escepticismo que en diversos filósofos del siglo XIV se desarrollarán de una manera diferente hasta la moderni$\mathrm{dad}^{4}$. La pérdida de la confianza en la capacidad cognoscitiva del intelecto huma-

3 R. Pasnau-J. Toivanen, "Peter John Olivi”, en E.N. Zalta (ed.), The Stanford Encyclopedia of Philosophy (Summer 2013 Edition) http://plato.stanford.edu/archives/sum2013/entries/olivi. La traducción es mía.

4 Cf. K. Michalski, Les sources du criticisme et du scepticisme dans la philosophie du XIVe siècle, Imprimerie de l'Université, Cracovie 1924, 2-3. No se olvide que uno de los rasgos que caracteriza el contexto de la filosofía cartesiana es precisamente el escepticismo, particularmente vigoroso en la Francia del Renacimiento, especialmente en Michel de Montaigne. Cf. L. Delia, La verità filosofica nel pensiero di Descartes: studio storico, critico e semantico (tesis doctoral), Bologna 2006, 97 quien, con expresión tomada de Brunschwigc, afirma que Descartes "fu anche un attento lettore degli Essais di Montaigne". L. Brunschwigc, en su estudio clásico, titulado Descartes et Pascal lecteurs de Montaigne (La Baconnière, Paris 1942, 9), sostenía que Montaigne fue "l'homme le plus marquant de la renaissance du scepticisme antique qui eut lieu au XVIe siècle". Sobre el renacimiento del escepticismo en la época moderna, cf. R. H. Popkin, Histoire du scepticisme d'Erasme à Spinoza (trad. fr.), PUF, Paris 1995. Cf. también Ch. Larmore, "Scepticism", en M. Ayers-D. Garber (eds.), The Cambridge History of Seventeenth Century Philosophy, Cambridge University Press, Cambridge 1998, vol. II, 1145-1192. Cf. igualmente el estudio de M. Burnyeat (ed.), The Skeptical Tradition, University of California Press, Berkeley 1983, así como también el de P.-F. Moreau (ed.), Le scepticisme au XVIe et au XVIIe siècle, Albin Michel, Paris 2001. 
no, uno de los caracteres distintivos de la filosofía del siglo XIV, hunde sus raíces también en la teoría agustianiana de la iluminación. Según san Agustín las rationes aeternae del intelecto divino resplandecen en el intelecto humano y crean en él su reflejo. Por ello, la certeza corresponde sólo a las ideas necesarias e inmutables, obtenidas únicamente gracias a la irradiación de la luz divina, no a la actividad del propio intelecto.

Pero, además de motivos de naturaleza teológica y del influjo de la noética de san Agustín, también existen motivos de naturaleza lógica que coadyuvarán a la difusión del escepticismo y del probabilismo en la baja Edad Media. La lógica del siglo XIV se asienta en buena medida sobre la distinción aristotélica entre dos formas de argumentación: una estricta, la demonstratio, presentada en los Analíticos segundos, y otra probable, la dialectica, expuesta en los Tópicos. Pues bien, en esta época la lógica se aparta de la demostración y se convierte cada vez más en dialéctica, es decir, en argumentación probable. El probabilismo se impone ante todo por el fuerte influjo de Pedro Hispano y de sus Summulae 5 . Así, en detrimento de la certeza fundada en la demostración, se asiste a lo largo del siglo XIV a la creciente importancia que en las disputas dialécticas adquirirá la probabilidad. Con frecuencia creciente la probabilidad pasa en las disputas al primer plano. Dice Michalski al respecto: "Las objeciones nacidas en las grandes discusiones públicas reducen al rango de puras probabilidades lo que hasta entonces había sido universalmente tenido como absolutamente cierto. Y, en sentido contrario, tesis hasta entonces consideradas evidentemente erróneas y falsas pasan a ser consideradas como probables cuando pueden ser fundadas en una argumentación cualquiera"6.

Pues bien, todos estas ideas se hallan presentes en los siglos de transición de la baja Edad Media a la modernidad. Como vamos a ver en seguida, en torno a ellas encontramos una estrecha vinculación entre tres autores paradigmáticos en la transición del tardomedievo a la modernidad, como son Ockham, Suárez y Descartes. La idea del concepto sin objeto se reforzará además con la doctrina del esse objectivum, que gozará de una larga acogida entre los filósofos y teólogos del siglo XIV, como son, entre otros: Ricardo de Mediavilla, Duns Escoto, Herveo Natalis, Pedro Aureolo, Durando de san Porciano y un largo etcétera. A su vez, la idea del esse

\footnotetext{
5 Hay que notar que las Summulae de Pedro Hispano no son tanto un manual de lógica, sino de dialéctica en el sentido estricto del término. Su cometido era preparar para las disputas dialécticas de la vida universitaria. Pedro Hispano afirma que sus discusiones no tienen más que un carácter de probabilidad. Los Analytica Posteriora, que forman la parte esencial de todo el Organon de Aristóteles,

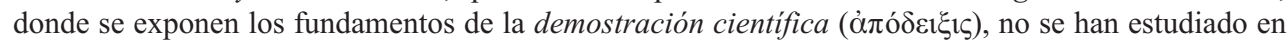
las Summulae. Por el contrario, el libro VI de las Summulae, que es el centro en el que converge el resto de la obra, está enteramente consagrado a los Tópicos, tal como fueron reelaborados por Boecio, y contiene todas las prescripciones de la lucha dialéctica. Por otro lado, Buridán en su reedición de las Summulae de Pedro Hispano ha identificado ya sin más la lógica y la dialéctica.

${ }^{6} \mathrm{~K}$. Michalski Les courants philosophiques à Oxford et à Paris pendant le XIVe siècle, Imprimerie de l’Université, Cracovie 1921, 6. La traducción es mía.
} 
objectivum será uno de los motivos de donde surja el fenomenismo de algunos autores modernos. De nuevo con Michalski: "Al problema de los universales se suma en el siglo XIV la cuestión de la diferencia entre concimiento intuitivo y abstracto. La teoría del esse objectivum, digámoslo desde ahora, ha conducido al fenomenismo, después de haber sido recibido como elemento constitutivo en el conceptualismo lógico"7. Más recientemente Pasnau ha empleado la expresión de "el velo de las species" para referirse a la dirección fenomenista emprendida por la teoría del conocimiento de algunos autores de la época que venimos estudiando: Enrique de Gante, Guillermo Crathorn, Pedro Olivi y Guillermo de Ockham 8 .

En breve, los cambios a los que se asiste en la metafísica y en la noética del siglo XIV van a favorecer una profunda transformación en las nociones de realidad y verdad, y en consecuencia, de sujeto y objeto de conocimiento.

\section{Avicena y los precedentes de la noción del esse objectivum: el tercer estado de la esencia}

La doctrina del esse objectivum forjada en el siglo XIV tiene un presupuesto metafísico fundamental: la noción aviceniana de la esencia. El mundo de Avicena es un mundo de esencias, que constituyen el objeto propio de su metafísica. Pero la metafísica de Avicena es peculiar en la misma medida en que es peculiar su concepción de la esencia.

La esencia cumple en la filosofía de Avicena dos funciones. En primer lugar, es el núcleo metafísico de estructuración de toda realidad. "Todo lo que es tiene una esencia que hace que sea lo que es, que posea el ser y que sea necesario"9. De estos tres aspectos enunciados, el primero es el formal o quiditativo; el segundo expresa la possibilitas ad esse y el tercero la necessitas ab alio. Así pues, la esencia se presenta en Avicena como el constitutivo formal de lo real y como el elemento metafísico primordial que permite existir, que no es sino recibir el ser de parte de un agente externo. Pero además de su función metafisica, la esencia desempeña también una función noética: es el principio de inteligibilidad de lo real. La aprehensión de la esencia es lo que permite el conocimiento de algo.

Sin embargo, aunque sean dos las funciones que desempeña la esencia, Avicena añade un tercer modo de considerar la esencia. En este sentido, Avicena es el primer pensador, históricamente hablando, en establecer una división tripartita de la

\footnotetext{
${ }^{7}$ K. Michalski, Les sources du criticisme..., 11. La traducción es mía.

8 Cf. R. Pasnau, Theories of cognition in the later Middle Ages, Cambridge University Press, Cambridge 1997, 220-253.

${ }_{9}$ Avicena, Logica, I, 3, en Id., Opera in lucem redacta ac nuper quantum ars niti potuit per canonicos emendata, Venetiis 1508. Reimpr. Minerva-Verlag, Frankfurt a. M. 1961.
} 
esencia. El texto fundamental al respecto se encuentra al inicio de su Lógica, donde se dice así: "Las esencias de las cosas o están en las mismas cosas o están en el intelecto. De ahí que tengan tres respectos: uno se da cuando la esencia en cuanto tal no es relativa a un tercer ser, ni a aquello que de ella se sigue por ser tal; otro aspecto es según como está en estos singulares; y el otro, según como está en el intelecto"10. La indicación resulta del máximo interés: la esencia se halla en dos lugares, pero admite tres respectos.

Desde luego, la esencia se da en dos lugares, por así decir, por lo que tiene sólo dos modos de estar: uno, realmente, en una cosa; otro, intencionalmente, en una inteligencia. Sin embargo, Avicena alude a un tercer modo de la esencia. No es, desde luego, un tercer modo real de la esencia, pues "todo lo que es o no existe en un sujeto o existe en un sujeto"11; es decir, o es un sujeto o no es un sujeto. Tertium non datur. No cabe un tercer modo de existir.

En efecto, la esencia se encuentra, inicialmente, en las cosas, de donde la abstrae el intelecto humano ${ }^{12}$. Sin embargo, tras admitir que la esencia sólo existe en la mente o en la cosa, Avicena considera la esencia en sí misma, como lo que tal esencia sería si no hubiese cosa ni inteligencia algunas. No se pretende de este modo investigar qué relación se da entre la esencia y el individuo, sino la consideración absoluta y aislada de la esencia. Por eso, en esta peculiar consideración de la esencia se prescinde de las condiciones con que ésta se presenta en el concepto (la universalidad) o en la cosa (la singularidad), para alcanzar así la esencia tal como es puramente, absolutamente, en sí misma. Pues bien, así considerada, la esencia es un puro eidós, una pura ratio, extraña y ajena a las propiedades que necesariamente ha de adquirir en su estado real: la particularidad real y la universalidad conceptual. Pero, considerada al margen de su relación con cosas y conceptos, la esencia es una pura esencia, una ratio equinitatis tantum. La esencia equinidad es simplemente equinidad (ipsa equinitas non est aliquid nisi equinitas tantum). Ciertamente la esencia se presenta realmente en dos estados. Pero en sí misma no es reductible a ninguno de ellos. La esencia considerada en sí misma goza de una prioridad sobre los otros estados, pues está totalmente separada del ser. Ni es en la cosa, ni en el concepto. Es tan sólo la identidad y su misma posibilidad. Posibilidad que es la ausencia de contradicción intrínseca, que puede ser cosa sin reclamar el

\footnotetext{
10 Avicena, Logica, I, 2: "Essentiae vero aut sunt in ipsis rebus aut sunt in intellectu; unde habent tres respectus. Unus respectus essentiae est secundum quod ipsa est non relata ad aliquod tertium esse, nec ad id quod sequitur eam secundum quod ipsa est sic. Alius respectus est secundum quod est in his singularibus. Et aliud secundum quod est in intellectu".

11 Avicena, Metaphysica, IV, 2, 85, en Opera in lucem.... "Omnis autem quae est, vel est existens non in subiecto, vel existens in subiecto".

12 Cf. Avicena, Metaphysica, V, 1, 87: "In intellectu enim est forma naturalis abstracta secundum abstractionem quam praediximus".
} 
ser ni oponerse a él como algo imposible, y que puede ser concepto sin exigir un intelecto que la piense ni se oponga a él como lo impensable.

La consideración de la esencia como un tertium genus, irreal y neutro, diferente tanto del ser real de la cosa como del ser intencional del concepto, fue prontamente acogida por Enrique de Gante, Escoto, Herveus Natalis, etc., y por medio de ellos pasó a la escolástica posterior (en especial a Suárez, con su noción de essentia realis) y al pensamiento moderno (Descartes, Leibniz, Wolff, Husserl, etc.) bajo diversos nombres: concepto objetivo, ente ideal, objeto puro, etc. 13 A veces esta esencia absoluta se ha dado en llamar universal ante rem o simplemente especie universal.

En cualquier caso interesa hacer notar que ya en Avicena aparece planteada la extraña modalidad de la esencia (después retomada por Escoto y los escolásticos tardíos), entendida como un esse objectivum, intermedio entre el ser real de la cosa y el ser psíquico del concepto, que no es ni individuo como aquél ni universal como éste. La esencia, así entendida, es una suerte de ser intermedio entre los dos términos referidos ${ }^{14}$.

\section{Un caso particular en la transición de la filosofía tardomedieval a la filoso- fía moderna: la relación entre las noéticas de Ockham, Suárez y Descartes}

A la luz de lo expuesto especialmente en el primer epígrafe, vamos a ver ahora brevemente cómo se entreteje una relación concreta entre tres autores, cronológicamente localizados en los siglos XIV, XVI y XVII como son, respectivamente, Ockham, Suárez y Descartes.

Según Ockham, no es contradictorio separar el acto de conocimiento (incluso el conocimiento intuitivo) de su objeto causante, poniendo en lugar de la cosa la intervención inmediata de la omnipotencia divina como causa primera. La razón de ello es que la causa primera, como fundamento de las causas segundas, puede realizar por sí misma lo que hacen éstas y de este modo reemplazarlas en su función causal. Ello vale tanto para la notitia intuitiva en particular, como a fortiori para todo el conocimiento humano en general. En virtud de esta doctrina, el conocimiento deja

\footnotetext{
13 Cf. A. García Marqués, "La polémica sobre el ser en el Avicena y Averroes latinos", en Anuario filosófico 1987 (20) 73-103, 77.

14 Avicena, Metaphysica, V, 1, 86: "Ergo universale ex hoc quod est universale est quiddam, et ex hoc quod est quiddam cui accidit universalitas est quiddam aliud [...] Definitio enim equinitatis est praeter definitionem universalitatis, nec universalitas continetur in definitione equinitatis. Equinitas enim habet definitionem quae non eget universalitate. Sed est cui accidit universalitas. Unde ipsa equinitas non est aliquid nisi equinitas tantum. Ipsa enim ex se nec est multa nec unum nec est existens in his sensibilibus nec in anima, nec est aliquod horum potentia vel effectu, ita ut contineatur intra essentiam equinitatis".
} 
de definirse formalmente por su relación natural y necesaria con la cosa. La intencionalidad del conocimiento queda irremediablemente problematizada, en la medida misma que se admite la posibilidad de un conocimiento intuitivo al que no corresponde cosa alguna existente.

Pero si, por otro lado, además de estimarse posible un conocimiento sin objeto, se acepta, como hace Ockham, la idea de la absoluta contingencia de las cosas creadas, de un lado, y la doctrina de la omnímoda soberanía de la potencia divina, de otro, ningún conocimiento humano podrá estar seguro de su verdad. El escepticismo resulta entonces inevitable. La omnipotencia divina juega aquí el papel de lo que posteriormente Descartes llamará el mauvais génie. Oigamos la suposición cartesiana del genio maligno: "Supponam igitur non optimum Deum, fontem veritatis, sed genium aliquem malignum, eundemque summe potentem et callidum, omnem suam industriam in eo posuisse, ut me falleret"15.

Pues bien, esta doctrina de un conocimiento objetivo sin objeto propio, cuya función causal es asumida por la omnipotencia divina, es explícitamente admitida por autores como Suárez, Fonseca y los Conimbricenses, cuyo influjo sobre Descartes y otros autores modernos nos es cada día mejor conocido.

En efecto, dos pasajes de Suárez nos ayudan a poner de manifiesto esta relación entre Ockham, Suárez y Descartes. El primero pertenece al Tractatus De Anima 3, 12, 5 y el segundo a los Commentaria una cum quaestionibus in libros Aristotelis De Anima, disp. 6, q. 5, n.6. En ellos se considera la posibilidad de que una determinada facultad cognoscitiva pueda conocer algo en ausencia de su objeto natural en virtud de la omnipotencia divina, lo cual haría sospechar que Dios induce al error al mover dicha facultad de conocimiento sin que a la misma corresponda objeto real alguno.

Hay que aclarar que los pasajes que presentamos a continuación son prácticamente idénticos porque pertenecen en realidad a una misma obra, aunque en distintas versiones. El primer texto corresponde a la paráfrasis de la obra de Suárez realizada por el teólogo conimbricense Baltasar Álvarez, que es la que ha venido a ser la edición príncipe del Tratado De Anima de Suárez (Lyon 1621), recogida posteriormente en la edición de L. Vivès. El segundo texto es el original de la misma obra, los Commentaria una cum quaestionibus in libros Aristotelis De Anima, publicada por primera vez por Salvador Castellote entre 1978 y 199116 . La parte del

15 R. Descartes, Meditationes de prima philosophia, I, AT VII, 22.

16 Cf. S. Castellote, Francisco Suárez De anima, 3 vol., Madrid 1978-1991, con traducción de C. Baciero y L. Baciero. El primer volumen se titula Francisco Suárez De Anima, Tomo 1, Texto inédito de los doce primeros capítulos. Facsímil de la segunda versión suareciana (Lyon 1621), Sociedad de 
manuscrito del De Anima corregida por el propio Suárez para la publicación de la edición de Lyon de 1621 llegó solamente a las disp. 2, q. 6 del original, que se corresponde con el lib. 1, cap. 12 de la edición de Lyon. Ello quiere decir que el texto más fiel al pensamiento de Suárez a partir del lib. 1, cap. 12 es el de los Commentaria una cum quaestionibus in libros Aristotelis De Anima. Por ello, el texto que ha de ser preferido es el segundo, que es el original. En cualquier caso, creo oportuno dejar constancia de ambos textos.

Dice en tal sentido el primer texto: "El sentido externo puede conocer la cosa ausente en virtud del poder divino. Tal cosa se prueba porque Dios puede conservar en el ojo la especie de la cosa ausente [...] Lo anterior consta por el hecho de que Dios puede suplir la función de la causa eficiente [...] Replicarás entonces diciendo que [en tal caso] Dios engañaría. Pero hay que negar tal cosa, porque únicamente permitiría el error en lo que se refiere a la presencia junto al cognoscente del objeto, no en lo relativo a la especie verdadera del objeto, que [Él mismo] pondría [immiteret] con su poder divino [divinitus] en el cognoscente"17.

estudios y publicaciones, Madrid 1978. El segundo, Francisco Suárez De Anima, Tomo 2 (d. 2 , q. 7 - d.7, q. 16), Editorial Labor, Madrid 1981. El tercero, finalmente, Francisco Suárez De Anima, Tomo 3 (d. 8 - d. 14), Fundación Xabier Zubiri, Madrid 1991. De la historia y los avatares del Tratado De Anima de Suárez da cuenta cumplida S. Castellote en el primer volumen de esta edición crítica. Sus hitos fundamentales son los siguientes. Suárez impartió una serie de cursos de filosofía entre 1571 y 1575. En 1572, a la edad de 25 años, tuvo un curso de filosofía en el que se incluyó el Tratado De Anima en forma de comentarios al Peri psychés de Aristóteles y que quedó inédito durante la vida de Suárez. Más adelante, poco antes de su muerte (Lisboa, 25 septiembre 1617), dedicó Suárez sus últimas fuerzas a la elaboración de un trabajo de psicología, que pudiera servir de fundamento de su teología, como ya había hecho con las Disputationes metaphysicae. En este trabajo Suárez comenzó retocando los tratados De angelis y el De opere sex dierum. Continuó posteriormente con la refundición del tratado De Anima, utilizando para ello el manuscrito mencionado de los años juveniles, pero sólo pudo hacer una nueva redacción de los doce primeros capítulos del libro primero de aquel Tratado $D e$ Anima. Como indica puntualmente Castellote "en esta refundición cambió Suárez la articulación del tratado, sustituyendo las disputationes del primitivo Manuscrito por libri, y las quaestiones por capita” (p. XL). Con carácter póstumo, cuatro años después de la muerte de Suárez, el P. Baltasar Álvarez publicó el Tratado De Anima en Lyon en 1621. El P. Álvarez se encontró, pues, con dos redacciones del Tratado De Anima: la primitiva y la refundida (de los doce primeros capítulos), optando "por publicar la versión nueva de los doce capítulos y el resto (“supplementum") de la antigua” (p. XL). De este modo había una redacción inédita (la primitiva de los doce primeros capítulos), y una edición, la del P. Álvarez, compuesta por la redacción nueva de los doce primeros capítulos y el resto de la primitiva. Ante este estado de cosas, además, S. Castellote nos informa de que en la elaboración de la edición crítica de este tratado se tuvo inevitablemente que proceder a un cotejo entre los Manuscritos antiguos del tratado De Anima y el texto de la edición príncipe hecha por Álvarez en Lyon 1621, del cual resultó "que no había una correspondencia exacta entre los manuscritos, por una parte, y la edición de Lyon, por otra" (p. XLI). En breve, Álvarez había manipulado los textos de Suárez. Precisamente las diferencias entre los dos textos mencionados se deben a la intervención del P. Álvarez en el pasaje del Tratado De Anima, lib. 3, cap. 12, 5.

17 F. Suárez, Tractatus De Anima, 3, 12, 5, en R.P. Francisci Suarez e Societate Jesu Opera omnia, vol. III, apud Ludovicum Vivès, Parisiis 1856, 657: "Sensus externus virtute divina cognoscere potest 
El segundo texto, que es, insistimos, el original de Suárez, dice así: "En el plano de la posibilidad absoluta [De potentia absoluta] es posible el conocimiento de un objeto ausente por los sentidos externos. Prueba: Dios es capaz de conservar en el ojo las especies de un objeto ausente y entonces el ojo vería por medio de ellas, a tenor de las afirmaciones anteriores. El antecedente es evidente: Dios puede suplir las veces de la causa eficiente. Ahora bien, las especies dependen del objeto únicamente en el género de lo eficiente [...] Una pregunta: la visión que nos ocupa, ¿sería intuitiva o abstractiva? Respuesta: En realidad sería verdaderamente abstractiva, por serlo de un objeto ausente, si bien la vista estimaría hallarse ante un objeto presente, puesto que lo vería del mismo modo que si el objeto estuviera presente. Una objeción: [Pero] En el [tal] caso, Dios sería autor del error. Respuesta: En absoluto; se limitaría a permitir el error del sentido, porque mientras Dios produce auténticas especies en el ojo, el hombre yerra al juzgar que el objeto le está presente"18.

Pues bien, en la primera parte de ambos textos aparece la idea tardomedieval, especialmente de origen ockhamista, de un conocimiento que, de potentia Dei absoluta, surge en la conciencia sin concurso de objeto real alguno; y en la segunda parte se anticipa la sospecha cartesiana del mauvais génie ${ }^{19}$. Como se ve, intencionalidad y certeza son puestas en cuestión.

rem absentem. Probatur: nam Deus conservare potest in oculo speciem rei absentis [...] Antecedens patet, quia Deus potest supplere vicem causae efficientis, species autem eo tantum modo pendet ab objecto [...] an vero talis visio intuitiva esset an abstractivam respondendum vere quidem esse abstractivam utpote rei absentis, videns tamen falso judicaret, ut praesentem, cum ita videret, ac si esset praesens. Urgebis, ergo jam tunc Deus deciperet. Negandum vero, sed permitteret tantum videntem falli, objectum namque veram sui speciem divinitus immiteret, videns autem seipsum falleret putandum objectum sibi adesse". La traducción es mía. También lo son las cursivas en el texto traducido.

18 F. Suárez, Commentaria una cum quaestionibus in libros Aristotelis De Anima, 6, 5, 6, en S. Castellote, Francisco Suárez De anima, Tomo II, Editorial Labor, Madrid 1981, 525: "De potentia absoluta fieri potest, ut per sensus externos cognoscatur res absens. Probatur, nam Deus potest conservare in oculo species rei absentis, et tunc oculus videret per illas, iuxta supra dicta. Antecedens patet, nam Deus potest supplere vicem causae efficientis; species autem tantum pendet ab obiecto in genere efficientis [...] Sed quaeres, an tunc illa visio esset intuitiva vel abstractiva. Respondetur, quod vere in re esset abstractiva, quia esset rei absentis, tamen visus iudicaret se habere rem praesentem, nam eodem modo videret ac si res esset praesens. Dices: Ergo tunc Deus falleret. Respondetur: Minime, sed permitteret tantum sensum falli, nam Deus veras species producit in oculo, ipse autem homo se fallit iudicans rem illam esse sibi praesentem". Las cursivas son mías.

19 Reproducimos aquí la versión francesa del texto de las Meditaciones, de la que ya hemos dado la versión latina. Cf. R. Descartes, Méditations touchant la première philosophie, I, AT. IXa 17: "Je supposerai donc qu'il y a, non point un vrai Dieu, qui est la souveraine source de vérité, mai un certain mauvais génie, non moins rusé et trompeur que puissant, qui a employé toute son industrie à me tromper". Además de los dos textos de Suárez ya citados, un tercero, tomado en esta ocasión de las Disputationes metaphysicae, presenta una idea semejante, aunque sólo en parte, a la de los textos anteriores. El nuevo texto al que nos referimos (DM 9, 2,7) se diferencia de los otros dos textos en que se refiere al intelecto, no al sentido, y, sobre todo, en que la temática está planteada en él de un modo bastante distinto a la de los dos pasajes anteriores. Con todo, la alusión que se encuentra en él al ángel malo, hace que el texto sea relevante para mostrar la continuidad histórica entre Suárez y Descartes. 
Estos textos nos ayudan a comprender cómo la noción de un concepto sin objeto, nacido en el contexto de la teología nominalista (Ockham), llegó en el siglo XVI hasta la filosofía de la segunda escolástica (Suárez), por medio de la cual desembocó en el pensamiento racionalista (Descartes), al que sugirió de este modo no sólo un nuevo método en la búsqueda de la verdad, sino un nuevo concepto de verdad.

Es verdad que en el segundo tercio del siglo XVII se asiste a diversos intentos de superación de la tesis del concepto sin objeto, el más importante de los cuales es probablemente el realizado por Juan de santo Tomás, quien en 1637 publica el Cursus philosophicus, obra de gran valor en el intento de renovar el realismo aristotélico. Sin embargo, según de Muralt, tal intento "llegó demasiado tarde para influir eficazmente en la historia de la filosofía moderna, porque, después de cuarenta años de sorprendente difusión de la obra metafísica de Suárez en Alemania, la noción de un conocimiento objetivo sin objeto había sido enseñada en todas las universidades luteranas, para acabar finalmente, tras una refundición completa, en la doctrina del fenómeno de Kant"20.

\footnotetext{
El texto en cuestión se encuentra en la DM 9, que se dedica al estudio de la falsedad (De falsitate seu falso). Se trata en concreto de la cuestión de si Dios o un ángel malo, como causas extrínsecas, pueden ser causa del error del intelecto. En concreto, Suárez plantea la cuestión de si Dios o un ángel malvado pueden obligar al intelecto proponiéndole algo falso ante lo que no pueda disentir. La cuestión es resuelta negativamente tanto en el caso de Dios como del ángel malo. En el primero se argumenta a partir de la bondad divina, a la que según la sana doctrina de los teólogos no repugna menos inducir al error [non minus inducere intellectum ad falsum] que mentir [quam mentiri]. En el segundo caso la posibilidad de que un ángel malvado sea la causa del error del intelecto es también rechazada, argumentando en un doble plano: primero, en el ejercicio del acto, y segundo, en su especificación. En el primer plano, el ángel malvado "no puede con su poder natural mover inmediatamente al intelecto al juicio o al acto segundo", porque tal poder "es propio de Dios, autor del mismo", y el carácter creatural del ángel le impide ser causa del acto del juicio. En el segundo plano, es decir, en el ámbito de la especificación del acto, con menos razón aún se puede admitir que el ángel malvado pueda obligar al intelecto al asentimiento de lo falso. Si acaso, dice Suárez, "puede inducir a lo sumo por medio de la sugestión o de la persuasión al asentimiento falso [...], pero siempre la cabe al hombre disentir, o al menos no asentir, si quiere". Para más información al respecto, cf. E. Faye, "Dieu trompeur, mauvais génie et origine de l'erreur selon Descartes et Suarez", en Revue philosophique de la France et de l'Étranger 126 (2001) 61-72; H. Gouhier, Le mauvais génie et le Bon Dieu. Essais sur Descartes, Vrin, Paris 1937 ; T. Gregory, "Dio ingannatore e genio maligno", en Giornale Critica della filosofia italiana 53 (1974) 477-516; N. J. Wells, "Material Falsity in Descartes, Arnauld, and Suarez", en Journal of the History of Philosophy 22 (1984) 25-50 ; E. Scribano, Guida alla lettura delle Meditazioni Metafisiche di Descartes, Laterza, Roma-Bari 1997; Id., "La nature du sujet, le doute et la conscience", en K.S. Ong-Van-Cung (ed.), Descartes et la question du sujet, PUF, Paris 1999, p. 4966 ; Id., "L'inganno divino nelle 'Meditazioni' di Descartes”, en Rivista di filosofia, 90/2 (1999) 219252.

20 A. de Muralt, "Epochè, Malin Génie, théologie de la toute-piussance divine: le concept objectif sans object. Recherche d'une structure de pensée", en Studia Philosophica 26 (1966) 159-191, 186.
} 


\section{Conceptualismo e inmanentismo tardomedieval}

Un rasgo común de la mayor parte de las teorías del conocimiento del siglo XIV es lo que con un término general se puede llamar conceptualismo. Dicho en breve y de un modo sencillo, el conceptualismo postula que el objeto de la inteligencia no es tanto la cosa misma, sino el concepto que el espíritu forma en sí mismo de ella. Como se puede ver, el conceptualismo supone un paso decisivo en el cuestionamiento de la intencionalidad del conocimiento y en el origen del principio de inmanencia. A ello se llega, como afirma De Wulf, bien porque se niega la correspondencia entre el concepto y la cosa o al menos porque se sostiene que no conocemos si hay correspondencia entre el concepto y la $\cos ^{21}$. De acuerdo con esta posición filosófica, el concepto no es tanto el acto de la mente que alcanza la cosa, sino el objeto mismo que ésta aprehende y en el que irremediablemente queda detenida ${ }^{22}$. Olivi afirma en este sentido que "las species tienen más razón de objeto que de principio intermedio [del conocimiento] o representativo" ${ }^{\text {"23. }}$.

Esta nueva modalidad del concepto en el que se conocen las cosas es el llamado conceptus objectivus y lo conocido en él es un esse objectivum. Se puede decir entonces que en el momento en que el concepto deja de ser entendido como mediación cognoscitiva y se convierte en el objeto mismo que se conoce y que, como tal, se pone ante el espíritu, la relación del pensamiento con la realidad de las cosas exteriores se vuelve problemática.

Esta interpretación del concepto como esse objectivum es consecuencia de la eliminación de la vieja noción de species. Ésta, perdida ahora su tradicional función de mediación cognoscitiva entre la cosa y el intelecto, es relegada a un segundo plano y sustituida por un tipo de repraesentatio cuya relación con la cosa conocida es difícil de determinar.

\footnotetext{
21 Cf. M. De Wulf, "Nominalism, Realism, Conceptualism”, en The Catholic Encyclopedia, vol. 11, Robert Appleton, New York 1913, 90.

22 R. Pasnau llama representacionismo (representationalism) al característico planteamiento inmanentista de no pocos filósofos tardomedievales que entienden la species no como aquello por medio de lo cual se conoce algo, sino aquello mismo que se conoce. Del representacionismo de Olivi afirma en concreto Pasnau: "The account Olivi rejects [...] that of Aquinas, for whom the species is an intermediary, a representation of the external object, and the source of cognition. Instead, Olivi concludes, the species will have to be the object of cognition, the thing cognized, not that by which one cognizes" (Theories of cognition in the later Middle Ages, 237).

23 Quaestiones in secundum librum Sententiarum, q. 58, ad 14, en "Bibliotheca Franciscana Scholastica medii aevi", vol. 5, ed. B. Jansen, Ad Claras Aquas [Quaracchi] prope Florentiam, ex typographia Collegii s. Bonaventurae 1924, 469: "Ergo species istae plus habebunt rationem obiecti quam rationem principii intermedii seu repraesentativi". Traducción y cursivas son mías.
} 
En el sentido clásico la species es la mediación entre la cosa y el entendimiento en cuanto hace presente al espíritu una cosa que en su estado natural le es exterior. Para Tomás de Aquino, por ejemplo, inspirándose ampliamente en Platón y Aristóteles, la species es la semejanza (similitudo) de la cosa, presente en el espíritu, y, en tal sentido, es repraesentatio. Pero se trata de una repraesentatio cuya relación con la cosa está bien establecida. Platón indica con el término eikasía la imagen que, en cuanto semejante a la cosa, se refleja en el alma ${ }^{24}$. Aristóteles precisa la naturaleza de la representación como acto de la imaginación (phantasia), "que es un movimiento producido por una sensación actual, y por eso tal movimiento es semejante a la sensación"25. De esta manera "los fantasmas [las imágenes] son semejanzas de los objetos sensibles separadas de la materia"26. En Aristóteles, pues, la representación es un contenido cognoscitivo de naturaleza sensorial, intermedio entre la percepción y la intelección y que, como semejanza de la cosa, cumple una función determinante en el proceso abstractivo del intelecto.

En esta tradición ininterrumpida que se remonta hasta Platón, Tomás de Aquino elabora una teoría general de la representación 27 . La representación es, pues, una semejanza de la cosa, a la que Tomás de Aquino suele llamar, con término de clara ascendencia griega, species. No se olvide que el término latino species traduce el término griego idea y no significa otra cosa sino aquella realidad esencial de la cosa que ésta transmite como información de sí a la facultad de conocimiento en cuestión. Inicialmente la especie es de índole sensible. Posteriormente, tras la acción universalizante del entendimiento, deviene inteligible. En cualquier caso, dada su naturaleza como semejanza de una cosa, la representación no es tanto la cosa misma conocida (sea sensitiva o intelectualmente), sino aquello en virtud de lo cual la cosa es alcanzada y conocida 28 . Por todo ello puede decirse que en sentido clásico la species procede de la cosa (por medio de la percepción sensible) y capacita al entendimiento para su retorno a ella.

La representación, en el sentido que este término adquiere tras Escoto, aparece como un esse objectivum, aislada en la conciencia, desprovista de toda señal que indique su procedencia de la cosa real y, en consecuencia, permita su reenvío a ella,

\footnotetext{
24 Cf. Platón, La República, 509d-511e.

25 Aristóteles, De anima, 428b 13-14.

26 Aristóteles, De anima, 432a 9-10.

27 Tomás de Aquino, De veritate, q.7, a.5, ad 2.

28 Cf. Tomás de Aquino, Summa Theologiae, I, q. 85, a. 2, co.
} 
o lo que es igual sin denotar su correspondencia con la cosa. Es verdad que el concepto sigue cumpliendo la función de procurar al espíritu un objectum, pero la relación de éste con la cosa real es, como mínimo, problemática. Desde luego, insistamos, esta representación no reenvía a la cosa misma. Permanece aprisionada en el intellectus. En la repraesentatio se opera en tal modo una reducción, que marcará profundamente a la filosofía moderna, de la res ipsa a su estado de esse cognita, o lo que es igual, de realitas objectiva. Esta reducción era favorecida claramente por las doctrinas -a las que nos referiremos después- de Escoto y Aureolo de una notitia abstractiva de la realidad ausente y, sobre todo, por la doctrina ockhamista de la posibilidad de un conocimiento intuitivo de una cosa no existente (notitia intuitiva rei non existentis).

Pero tras la posibilidad sugerida por Ockham de un conocimiento intuitivo de un objeto inexistente se encuentra un nuevo modo de entender la naturaleza de la realidad, y en definitiva, una nueva ratio realitatis. El ente, en esta perspectiva, ha devenido un objeto, cuya realidad es accesible al espíritu con independencia de su existencia. Ello quiere decir que el ente está perdiendo su contenido positivo y absoluto y está comenzando a ser considerado como algo que pertenece al espíritu, como un contenido o un momento del espíritu. Ahora bien, de este modo, absorbida la res en el intelecto, cambia también la relación del espíritu con la realidad, es decir, cambia la noción de verdad (como se ve de un modo particularmente claro en Aureolo), porque de ser determinado el espíritu humano pasa ahora a ser determinante. Sería excesivo decir que el giro copernicano anunciado por Kant en la Crítica de la razón pura tiene lugar en la baja Edad Media. Pero es claro que la fórmula de Aureolo, que veremos después, lo sugiere. En cualquier caso lo que de este modo se está transformando en la historia del pensamiento son los conceptos mismos de realidad y de verdad, o si se prefiere, está cambiando el modo de entender la relación del espiritu con la realidad.

En definitiva, un nuevo tipo de ser, como hemos visto en el epígrafe segundo, una suerte de intermediario ontológicamente neutro entre la res extra animam y la idea como acto psíquico del intelecto ha venido a colocarse entre los términos del problema: es la realitas objectiva o el esse objectivum in anima. Descartes la llamará más adelante la realitas objectiva de la cogitatio, o más propiamente, el cogitatum. La doctrina del esse objectivum, en realidad, se remonta, como ya se ha visto, a Avicena y Enrique de Gante, quienes habían considerado un peculiar modo de la esencia, distinto del ser real (individual) y del ser intencional (universal): la equinitas tantum. Durando de san Porciano ha expresado claramente la idea ya implícita en Escoto, según la cual el concepto objetivo no es simplemente un aspecto puramente exterior de la cosa misma -su denominación extrínseca, en cuanto conocidasino que es un verdadero tertium quid, que, aunque no tiene más existencia que la de un ens diminutum, le es suficiente para colocarse como una pantalla entre el acto 
psíquico del conocimiento (el llamado concepto formal) y la cosa en su existencia exterior ${ }^{29}$.

Esta nueva noética será, a su vez, el fundamento imprescindible para entender el sentido de la doctrina suareciana de la essentia realis. La esencia es entendida como un puro posible y simple cogitable. Tal esencia procura una base suficiente para la ciencia, incluso en un mundo en el que la existencia se diluyera. Un momento importante en este itinerario intelectual es Enrique de Gante, quien inspirándose en Avicena, define el esse proprium de la esencia al margen de la existencia actual, considerando así que la esencia es capaz de asegurar su realidad objetiva independientemente de su actualidad en el mundo de lo existente efectivamente. Este esse proprium de la esencia es el esse essentiae. Precisamente es Enrique de Gante el primer autor en emplear esta expresión, distinguiéndola de la de esse existentiae.

Queda entonces por determinar el grado de realidad que pertenece a este esse essentiae antes de ser actualizado por la existencia y de convertirse en una essentia actualis. El esse essentiae no es ciertamente un puro nihil, aunque comparado con el esse existentiae se muestre como debilius y como un ens diminutum. Se trata de la cuestión que Suárez afronta con el título de Quid sit essentia creaturae priusquam a Deo producatur ${ }^{30}$. Suárez identifica el esse essentiae con el esse reale, el cual, a su vez, es interpretado como el esse objectivum; o en otros términos, el ente se reduce a la esencia y ésta al concepto objetivo ${ }^{31}$.

29 Cf. R. Dalbiez, "Les sources scolastiques de la théorie cartésienne de l'être objectif", en Revue d'histoire de la philosophie 3 (1929) 464-472. Cf. también T. Cronin, Objective being in Descartes and in Suárez, Gregorian University Press, Roma 1966, especialmente 200-207.

30 Cf. DM, 31, 2, 1.

31 Para Suárez el ente es, ante todo, esencia real. Esta esencia real es meramente posible, si no existe, y actual si existe de hecho. Así el ente, reducido por Suárez a esencia real, no se dice real por existir, sino por tener la aptitud para existir. El ser real (esse reale), pues, no es según Suárez lo que existe, sino lo que, no siendo contradictorio, puede existir. El principio fundante del ente es, pues, la no contradictoriedad de su esencia y, en último análisis, la posibilidad lógica. Ahora bien, la evolución de la filosofía racionalista (Descartes, Clauberg, Leibniz, Wolff, Baumgarten Kant, etc.), tan fuertemente vinculada a Escoto y Suárez, demuestra finalmente que la posibilidad será resuelta en la pensabilidad del ente. Si para Suárez el ente no es "lo que es", sino "lo que puede ser", no debe extrañarnos que en la escuela postcartesiana del ocasionalismo, alimentada a fortiori de filosofía de la interioridad agustiniana, el ente venga a ser ya en Clauberg "lo que puede ser pensado" (id quod cogitari potest). Así, igualmente, en Wolff, Baumgarten, Kant, etc. Cf. L. Prieto, "Res, aliquid y nihil en Suárez y la filosofía moderna", en Anales del Seminario de Historia de la Filosofía, 30/1 (2013) 49-69, especialmente 61-69. En breve, estando así las cosas, el ente puede hacerse objeto del pensamiento, porque: $1^{\circ}$ ) es algo, en cuanto contradistinto de la nada, como significa el nombre aliquid; $2^{\circ}$ ) tiene un contenido quiditativo propio (significado por el nombre res) suficiente para el conocimiento, en virtud del cual es real, aunque no exista. Así entendido, el ente se convierte en un posible primero, en un cogitable después y finalmente viene a ser el dominio sin residuos de la razón. He aquí uno de los itinerarios intelectuales que han conformado la filosofía moderna y han propiciado la asunción del principio de inmanencia del conocimiento. 
Como se ve, lo que está en juego es la intencionalidad del conocimiento. Perdida o puesta en un segundo plano su vinculación con la cosa real exterior (como consecuencia de la pérdida de la colaboración de conocimiento sensible e intelectual y de la doctrina de las abstracción), la species deja de ser aquello por medio de lo cual se conoce la cosa para pasar a ser la cosa misma conocida. La ratio realitatis se desplaza así al interior del espíritu y la verdad queda dispensada del movimiento de salida a la realidad misma. Es lo que venimos llamando el principio de inmanencia.

En este sentido Dalbiez ha mostrado oportunamente que la cuestión sobre la naturaleza de la verdad fue debatida en el siglo XVI por Francisco Suárez y Gabriel Vásquez. Aunque con diferencias en la solución, dicha cuestión quedó planteada en los términos de una relación existente entre la cosa exterior, la species como dimensión psíquica del concepto (concepto formal) y el concepto objetivo ${ }^{32}$. Este modo de entender la naturaleza de la verdad llegó a La Flèche, donde Descartes pudo muy bien haber tenido conocimiento de ella 33 .

\section{Escoto, Aureolo y Ockham sobre el conocimiento}

Junto con la noción del esse objectivum inspirada en Avicena, las doctrinas que han conducido al conceptualismo característico del siglo XIV, son, entre otras, la distinción entre conocimiento abstractivo e intuitivo, la teología de la omnipotencia divina aplicada al conocimiento humano y la doctrina de la intuición de un objeto inexistente. Ya nos hemos referido a ellas en parte, pero de un modo insuficiente

\footnotetext{
32 Soncinas propone en sus Quaestiones metaphysicales acutissimae (que son un comentario a la Metafísica de Aristóteles) una noción de concepto formal y concepto objetivo que puede ser útil a nuestro propósito. El concepto formal es el actus intelligendi; el concepto objetivo es la res ipsa quae intelligitur. El texto dice así: "Primum est quod conceptus est duplex, scilicet formalis et obiectivus. Conceptus formalis est secundum aliquos actus intelligendi, et secundum S. Thomam est verum formatum de re per actum intelligendi. Conceptus obiectivus est res ipsa, quae actu vel potentia intelligitur. Quod enim res ad extra prout habet esse in intellectu, appelletur conceptus patet ex Averroë, dicente 3. de anima comm. 18, quod intentiones intellectae, continuantur cum intentionibus imaginatis: constat autem quod intentiones intellectae, sunt ipsae naturae, quae intelliguntur" (Quaestiones metaphysicales acutissimae, sumptibus Petri Landry, Lugduni 1579, q. 2, p. 2a-b). Cf. A. Contat, La relation de vérité chez saint Thomas, Libreria editrice Vaticana, Città del Vaticano 1996, 96-97, nota 50, donde el autor observa agudamente: "Le concept objectif, c'est ici la chose même, selon qu'elle a un esse dans l'intellect: cette définition manifeste bien la difficulté de cerner le défini, qui est à la fois dans la chose en tant qu'objet, et dans l'intellect en tant que concept'. Las cursivas, tanto del texto latino como francés, son mías.

33 Cf. R. Dalbiez, "Les sources scolastiques de la théorie cartésienne de l'être objectif", 467.
} 
que ahora completamos. La primera de ellas tiene su origen en Escoto; la segunda, está especialmente presente en Pedro Aureolo; la tercera, es una aportación característica de Guillermo de Ockham. Las tres doctrinas pertenecen a autores franciscanos, en cuyo pensamiento se ha renovado el agustinismo de los siglos precedentes y perdura vivo el eco de las condenas de París y Oxford de fines del siglo XIII.

\section{a) La doctrina de Escoto sobre la notitia intuitiva y la notitia asbtractiva}

La distinción escotista entre notitia abstractiva y notitia intuitiva es el punto de partida fundamental de las teorías del conocimiento tardomedievales.

Escoto construye su teoría del conocimiento sobre las nociones de abstracción e intuición. Entiende la abstracción como el conocimiento de la cosa real en cuanto ausente, pero suplida su ausencia por una repraesentatio. La intuición, en cambio, es entendida como el conocimiento de una cosa en cuanto inmediatamente dada y presente. Además de la diferencia en lo relativo al conocimiento intuitivo, salta a la vista la diferencia sobre la abstracción entre la noción escotista y la noción de Tomás de Aquino.

En realidad, la historia del pensamiento indica que el rechazo de la abstracción se basa en equívocos que se arrastran en la filosofía europea desde la crisis nominalista e incluso desde la sustitución por obra de Escoto de la aristotélica distinctio rationis cum fundamento in re por la distinctio formalis a parte rei34. En efecto, el sentido que Duns Escoto da a la noción de abstracción poco tiene que ver con el sentido clásico de abstracción como separación (abs-trahere) de una forma (portadora de la actualidad y, por tanto, de inteligibilidad) de su respectiva materia (como contenido potencial, inaferrable, en consecuencia, para la inteligencia). Escoto, por su parte, entiende la abstracción como referida a la existencia y presencia de una cosa. Así entendida, la abstracción es el procedimiento por el cual el cognoscente, dejando de lado la presencia del objeto, lo considera con independencia de su existencia o de su ser efectivamente dado. Como se ve, la noción de abstracción de Escoto prepara el terreno a la doctrina de la esencia como esse objectivum en cuanto objeto propio del conocimiento humano.

La notitia abstractiva, pues, se caracteriza por prescindir de la presencia del objeto, considerándolo con independencia (indiferentemente, dice Escoto) de la existencia (o presencia ante el cognoscente) que dicha res puede tener extra animam. Ello significa que el objeto de este acto de conocimiento, haciendo abstracción tanto de la presencia como de la ausencia de la cosa real conocida, sugiere un nuevo concepto de realidad, que, entendida en el sentido de esencia o quididad, deja de lado la existencia. Como acabamos de comentar, esta idea había sido suge-

34 Cf. A. Llano, Metafisica tras el final de la metafisica, Cristiandad, Madrid 2007, 88. 
rida por Avicena al considerar la esencia en sí misma, con distinción de su estado real en la cosa o intencional en el concepto.

La segunda modalidad de conocimiento, llamada notitia intuitiva, es presentada por Escoto como el acto de aprehensión inmediata del objeto ut existentis et praesentis. Dice así nuestro autor: "Hay otro acto del entendimiento, que sin embargo no experimentamos con igual grado de certeza en nosotros. Se trata de aquel acto que capta propiamente [praecise] el objeto presente en cuanto presente y el existente en cuanto existente. Este tipo de intelección se puede llamar intuitiva, porque es una intuición de la cosa en cuanto existente y presente"35.

En cualquier caso, el paso decisivo de esta doctrina del conocimiento está en la distinción entre el objectum in se praesens y la repraesentatio objecti per speciem, come se expresa claramente en el siguiente texto de Escoto: "La diferencia entre conocimiento intuitivo y abstractivo consiste en que éste, que es por medio de la species, no es de la cosa en cuanto existente ni presente, sino que puede, indiferentemente, ser de la cosa ausente y presente, porque en la especie se representa el objeto en cuanto manifiesto [ut relucet]; en cambio, el conocimiento intuitivo es de la cosa existente en cuanto existente" 36 .

\section{b) Aureolo, la teología de la omnipotencia divina}

Pedro Aureolo, teólogo y filósofo franciscano como Escoto, sigue los pasos de éste, a la vez que acentúa la presencia de motivos nominalistas, todavía incipientes en Escoto.

Como era de esperar, la teoría del conocimiento de Aureolo se inicia con la distinción entre notitia intuitiva y notitia asbtractiva. "Es, pues, una noticia intuitiva aquélla que se refiere a la existencia y presencia de la cosa y que termina en la cosa como es existente en sí. La abstracción, en cambio, se dice tal porque abstrae del ser y del no ser, del existir y no existir y de la presencialidad [del objeto]. De manera que intuyo la rosa cuando la concibo como presente, mientras que la concibo abstractamente cuando considero su quididad o naturaleza" 37 .

35 Escoto, Quaestiones quodlibetales, VI, n. 8, en Joannis Duns Scoti Opera omnia, XII (ed. Lucas Wadding, Lyon 1639), 145: "Alius autem actus intellligendi est, quem tamen non ita certitudinaliter experimur in nobis; possibilis tamen est talis, qui scilicet praecise sit objecti praesentis ut praesentis, et existentis ut existentis [...] ista inquam intellectio potest proprie dici intuitiva, quia ipsa est intuitio rei, ut existentis et praesentis". La traducción es mía.

36 Escoto, Collationes, XXXVI, n. 11, en Joannis Duns Scoti Opera omnia, III, 423: "Differentia est inter cognitionem intuitivam et abstractivam, quod cognitio abstractiva, quae est per speciem, non est rei ut existens, nec ut praesens est, sed indifferenter potest esse rei absentis et praesentis, sicut est species in qua repraesentaur objectum ut relucet; sed cognitio intuitiva est rei existentis, ut existentis est". La traducción y las cursivas son mías.

37 Aureolo, Scriptum super primum Sententiarum, proemium, sect. 2, art. 2, n. 52, en E. M. Buytaert (ed.), Petri Aureoli Scriptum super primum Sententiarum, I (Franciscan Institute Publications, Nueva 
Pero, a diferencia de Escoto, Aureolo añade una referencia a la potentia Dei absoluta, que es de la mayor importancia para la adecuada comprensión de la sensibilidad filosófica que se está gestando en el siglo XIV. Según Aureolo, la causa eficiente de la intelección puede ser no sólo la esencia de una cosa presente (como en el caso del conocimiento intuitivo), sino también la omnipotente voluntad divi$n a$, aunque tal notitia no termine en la esencia ut existentem et praesentem, y, por tanto, sea una noticia no intuitiva, sino abstractiva.

Dice Aureolo en concreto: "Dios puede hacer por su sola voluntad lo que puede hacer mediante su esencia. Pero mediante su esencia mueve el intelecto de los beatos al conocimiento claro y desnudo de Sí, que es intuitivo, en cuanto [este conocimiento] termina en ella [la esencia divina], porque lo mueve en cuanto presente realmente y existente. Luego por su sola voluntad Dios podría mover el intelecto al conocimiento claro y desnudo de su esencia. Es verdad que tal conocimiento termina en la divina esencia bajo aquel aspecto en que el intelecto es movido a ella. Pero no es movido por la presencialidad y existencia de la divina esencia, sino por imperio de su voluntad omnipotente. Por tanto, como quiera que tal conocimiento no termina en la esencia [divina] en cuanto presente y existente, sino en la esencia, pero abstrayendo simplemente de la existencia y de la presencialidad, en consecuencia tal conocimiento no será intuitivo, sino más bien abstractivo"38.

En cualquier caso, lo que sobre todo interesa constatar aquí es que, según Aureolo, la intervención de la omnipotencia divina puede dispensar al conocimiento de la presencia y de la existencia misma del objeto. En otros términos, la voluntad divina puede mover el intelecto sin que a éste corresponda objeto alguno. Como se ha sugerido antes, la omnipotencia divina puede proveer al conocimiento de un concepto sin objeto.

La teoría del conocimiento de Aureolo va así a conducir a un concepto de verdad que, en la medida de su origen agustiniano, será el inverso de la noción propuesta por Tomás de Aquino en su célebre fórmula del De Veritate. Para Aureolo,

York 1952) 191: "Est enim intuitiva notitia, quae concernit rei praesentialitatem et existentiam, et terminatur ad rem ut in se existentem. Abstractio vero dicitur quae abstrahit ab esse et non esse, existere et non existere, et a praesentialite; quemadmdum rosam intueor dum eam praesentem concipio, abstractive vero dum ejus quidditatem et naturam considero". Las cursivas son mías.

38 Cf. Aureolo, Scriptum super primum Sententiarum, proemium, sect. 2, art. 2, n. 53, en E. M. Buytaert (ed.), Petri Aureoli Scriptum super primum Sententiarum, I, 191: "Deus potest facere sola voluntate, quidquid facere potest mediante sua essentia; sed mediante essentia movet intellectum beati ad suam notitiam claram et nudam, quae quidam est intuitiva, pro eo quod terminatur ad eam, ut praesentem realiter et existentem, quoniam ut sic movet. Ergo sola voluntate poterit movere intellectum ad notitiam suae essentiae nudae et clarae. Certum est enim quod talis notitia sub illa ratione terminatur ad divinam essentiam, sub qua ratione intellectus movetur ad eam; non movetur autem per praesentialitatem et existentiam essentiae divinae, sed per imperium onnipotentis voluntatis. Ergo nec terminabitur talis notitia ad essentiam ut existentem et praesentem, sed ad essentiam mere abstrahendo ab existentia et prasentialitate et per consequens non erit intuitiva, sed potius asbtractiva". La traducción y las cursivas son mías. 
dice Vignaux, la verdad no consiste en una conformidad del espíritu con lo real, sino a la inversa: de lo real con el espíritu. Así pues, las cosas se dicen verdaderas en la medida que son fieles a su naturaleza, que existe solamente en el pensamiento 39 .

Aureolo razona del siguiente modo: la verdad de una cosa no es otra que su esencia, de manera que ser verdadero consiste en seguir su propia esencia ${ }^{40}$. Ahora, ninguna esencia es subsistente, porque las cosas creadas son imitaciones, reducciones (entidades disminuidas, entitates diminutae) de la esencia divina. Nuestros conceptos, pues, son verdaderos en la medida que imitan la esencia suprema, donde descansan los ejemplares de todas las cosas. De este modo Aureolo, como antes había hecho Escoto, retorna a san Agustín, privilegiando una noción de verdad de índole teológica.

\section{c) Ockham, la omnipotencia divina y la intuición del objeto no existente}

El descubrimiento de Escoto de la objetividad del conocimiento alcanzado por la notitia abstractiva y la doctrina de Aureolo de la omnipotencia divina capaz de originar en el cognoscente un concepto sin objeto son los precedentes inmediatos de la teoría del conocimiento de Ockham.

Detengámonos ahora un momento en la teoría del conocimiento de Ockham. Lo primero que hay que poner de manifiesto es que su filosofía es una filosofía del creyente. Es una reivindicación de una teología cristiana que quiere restablecer la soberanía y libertad divinas que el aristotelismo, especialmente el averroísmo árabe y latino, habían negado con su necesitarismo ontológico.

En este contexto es oportuno preguntarse, supuesta la omnipotencia divina, por el conocimiento humano ${ }^{41}$. Como Aureolo antes, también Ockham parte de Escoto. Nuestro conocimiento aprehende la cosa individual dada en primer lugar al sentido. Este conocimiento es la notitia intuitiva, cuyo objeto es lo que existe realmente y nos es presente. Ahora bien, ¿cómo ha de entenderse el conocimiento intuitivo cuando se cae en la cuenta de que todas las cosas son obra de un Dios libre, soberano y omnipotente? La fe en un Dios omnipotente conduce a Ockham a los dos siguientes principios:

a) Como Causa primera, Dios puede realizar por sí mismo lo que realiza por medio de las causas segundas. Dice Ockham al respecto: "En aquel artículo, a saber,

\footnotetext{
39 Cf. P. Vignaux, El pensamiento en la Edad Media, FCE, Madrid 1999, 157.

40 Cf. Aureolo, Scriptum super primum Sententiarum, dist. XIX, tertia pars, a. 2, en Commentariorum in primum librum Sententiarum, pars prima, ex typographia vaticana, Romae 1596, 494: "Veritas rei nihil aliud est quam eius quidditas, nec esse verum est aliud quam assequi propriam quidditatem [...] Nulla autem quidditas est subsistens, nec esse habens nisi in intellectu obiective, propter quod nulla veritas pura, et sincera est nisi in intelectu obiective, excepta prima veritate". Las cursivas son mías. 41 Cf. P. Vignaux, "Nominalisme", en Dictionnaire de Théologie Catholique, XI, Letouzey et Ané, Paris 1931, coll. 767-768.
} 
Creo en Dios Padre omnipotente, tiene su fundamento la famosa proposición de los teólogos: lo que Dios produce por medio de causas segundas, puede producirlo y conservarlo inmediatamente sin ellas" 42 . Igualmente: "Todo efecto que Dios puede [producir] mediante una causa segunda, puede [producirlo] inmediatamente por sí mismo"43. En definitiva, poseyendo Dios la plenitud de la potencia, no tiene necesidad de la mediación de las causas segundas.

b) Si dos cosas no existen ni en el mismo sujeto ni en el mismo lugar, Dios puede hacer existir la una sin la otra.

De la aplicación de estos principios al conocimiento intuitivo resulta lo siguiente: primero, como Causa primera del objeto que causa el conocimiento, Dios puede causar directa e inmediatamente el conocimiento en el sujeto cognoscente sin la mediación de la cosa; segundo, como el conocimiento, que es una cualidad del alma, y el objeto que lo causa, son dos cosas distintas, el primero puede existir sin el segundo.

Se llega así a la noción de la intuición del objeto no existente. ¿Puede darse conocimiento intuitivo de un objeto que no existe, en virtud del cual el espíritu conozca con evidencia este objeto no existente? La respuesta de Ockham es afirmativa. En efecto, "la noticia intuitiva, tanto sensitiva como intelectiva, puede ser de la cosa no existente" 44 . Ockham razona pasando del objeto existente y presente al objeto simplemente existente, pero ausente y de ahí, finalmente, al objeto no existente en un movimiento de transición de la eficiencia del objeto (causa segunda) sobre el sujeto cognoscente a la eficiencia inmediata de la omnipotente voluntad divina (Causa primera) sin la existencia del objeto. Con la intervención de la omnipotencia divina, el conocimiento intuitivo alcanza lo que no existe. En definitiva, no es contradictorio, según Ockham, separar el conocimiento intuitivo de su objeto

\footnotetext{
42 G. de Occam, Quodlibeta septem, VI, q. 6 (Utrum cognitio intuitiva possit esse de obiecto non existente), en Opera philosophica et theologica ad fidem codicum manuscriptorum edita: opera theologica Guillelmi de Ockham, IX, ed. J.C. Wey, Franciscan Institute Publications-St. Bonaventure University [NY] 1980, 604-605: "In illo articulo (sc. Credo in Deum Patrem omnipotentem) fundatur illa propositio famosa theologorum: Quicquid Deus producit mediantibus causis secundis, potest immediate sine illis producere et conservare". La traducción es mía. Cf. G. de Occam, Quodlibeta septem,VI, q. 6, en Opera philosophica et theologica ad fidem codicum manuscriptorum edita: opera theologica Guillelmi de Ockham, IX, 604: "In ista quaestione pono duas conclusiones: prima est quod cognitio intuitiva potest esse per potentiam divinam de obiecto non existente. Quod probo primum per articulum fidei: Credo in Deum Patrem omnipotentem".

43 G. de Occam, Quodlibeta septem, VI, q. 6, en Opera philosophica et theologica ad fidem codicum manuscriptorum edita: opera theologica Guillelmi de Ockham, IX, 605 : "Omnem effectum quem potest Deus mediante causa secunda, potest immediate per se". La traducción es mía.

44 Ockham, In I Sent., prol., q. 1, a. 1, en Opera philosophica et theologica ad fidem codicum manuscriptorum edita: opera theologica Guillelmi de Ockham, I, ed. G. Gál-S.F. Brown, Franciscan Institute Publications-St. Bonaventure University [NY] 1967, 38: "Intuitiva notitia, tam sensitiva quam intellectiva, potest esse de re non existente".
} 
propio, poniendo en su lugar la intervención inmediata de la Causa primera. Como fundamento de las causas segundas, la Causa primera puede suplir la eficacia de las criaturas sobre el cognoscente. Ello vale para la notitia intuitiva en particular, así como para todo el conocimiento humano en general.

El conocimiento deja entonces de definirse formalmente por su relación natural y necesaria con la cosa. De este modo, la intencionalidad del conocimiento se pierde, si es en verdad posible que se dé un conocimiento intuitivo de un objeto inexistente. Pero no sólo la intencionalidad queda en entredicho, también la certeza del conocimiento. Así como es posible un conocimiento sin objeto, así también, en razón de la absoluta contingencia de las cosas creadas, o lo que es igual, en virtud de la soberanía absoluta de la omnipotencia divina, ningún conocimiento humano puede estar seguro de su verdad. Como hemos dicho antes, la omnipotencia divina juega aquí el papel del malin génie cartesiano. Intencionalidad y certeza quedan cuestionadas o negadas en esta filosofía que admite la posibilidad de un conocimiento intuitivo sin objeto propio. Dicho de otro modo, el criticismo y el escepticismo reciben un impulso decisivo de la teoría del conocimiento de Ockham.

\section{Conclusión}

En esta investigación hemos estudiado algunas de las principales ideas de las teorías del conocimiento tardomedievales, especialmente del siglo XIV, con el propósito de conocer con más detalle las razones del surgimiento del nuevo paradigma del conocimiento, el principio de inmanencia, asumido por la mayor parte de los filósofos modernos.

Constatándose en este trabajo la continuidad entre la historia de la filosofía tardomedieval y moderna, resulta insostenible la tesis de una Edad Media intelectualmente irrelevante y de un surgimiento ex novo del pensamiento moderno. Diversos trabajos del siglo pasado dieron cuenta suficientemente del hecho de la procedencia medieval de no pocas ideas de la filosofía moderna. Para mencionar sólo algunos ejemplos, citemos los nombres de Freudentahl (aunque su trabajo es de finales del siglo XIX), Gilson y Krakowski, que dedicaron relevantes estudios a las fuentes medievales y escolásticas del pensamiento de Spinoza, Descartes y Locke, respectivamente $^{45}$. También Michalski, ya citado al inicio de este artículo, estudió por aquella época algunas ideas de la lógica y de la teoría del conocimiento de los últimos siglos de la Edad Media, poniendo de manifiesto en diversos trabajos el influ-

45 Cf. J. Freundentahl, "Spinoza und die Scholastik", en F. Vischer (ed.), Philophiche Aufsätze, Eduard Zeller gewidmet, Fues's Verlag, Leizig 1887, 83-138; E. Gilson, Index scolastico-cartésien, Felix Alcan, Paris 1913; E. Krakowski, Les sources médiévales de la philosophie de Locke, Jouve at Cie editeurs, Paris 1915. 
jo que algunas doctrinas tardomedievales (particularmente el escepticismo y el criticismo) habían tenido sobre determinadas corrientes del pensamiento moderno.

Precisamente en el característico criticismo de la filosofía tardomedieval, sobre todo de Escoto en adelante, se percibe el progresivo afianzamiento del principio de inmanencia del conocimiento, un principio de importancia decisiva en el origen y desarrollo de la filosofía moderna, tal como se descubre fácilmente en figuras tan relevantes como Descartes, Locke y Kant, para referirnos sólo a tres grandes maestros de la filosofía moderna. La pérdida de la confianza en el conocimiento humano que acompaña a buena parte de los autores de la baja Edad Media, manifestada en las múltiples formas de escepticismo gnoseológico, voluntarismo ético y fideísmo teológico, llevará a la filosofía a hacerse cuestión no tanto de las cosas, sino del hecho mismo del conocimiento. El criticismo siempre ha sido precedido de alguna forma de escepticismo. La relevancia extraordinaria que en los filósofos y teólogos del siglo XIV reviste la teoría del conocimiento es un indicio inequívoco del giro crítico que se está produciendo. Poco a poco, desde entonces, la filosofía cambia el objeto de su actividad, pasando de la investigación de la cosa real (exterior) a la de la cosa conocida (concepto o idea).

Como se ha dicho al inicio, la doctrina escotista de la notitia abstractiva, que alcanzó una rápida difusión entre todos los pensadores del siglo XIV, contribuyó a elaborar una teoría de la representación cuya relación con la realidad natural era no sólo débil, sino problemática. De ahí que se entienda que el verdadero objeto del espíritu no sea la cosa, sino aquel objeto inmanente al propio espíritu puesto en él por la repraesentatio: el esse objectivum del concepto. Algunas ideas teológicas contribuirán además en este proceso. El voluntarismo admite que, en virtud de la omnipotencia y libertad divinas, es posible un acto de conocimiento sin correspondencia con cosa alguna. Siendo Dios la omnipotente Causa primera, podría tomar, de potentia absoluta, el lugar de las cosas en el origen del conocimiento y venir a ser Él mismo la causa inmediata de un acto de conocimiento que no tiene correlato con ninguna realidad exterior. Ahora bien, llegados a este punto, se estimará que sólo el recurso a la bondad divina puede evitar -y está por ver si efectivamente lo logra- la conclusión de considerar a Dios como engañador, o al menos, como inductor al error, como encontramos en diversos pasajes de la obra de Suárez y Descartes. En realidad, en descargo de Descartes hay que decir que los teólogos calvinistas de Leyden (Triglandius y Revius) que lo acusaron de blasfemia por el empleo de la hipótesis del genio maligno desconocían la larga historia que dicha idea arrastraba desde el siglo XIV46.

46 En 1647 un profesor de la Universidad de Leyden, el teólogo calvinista Revius, acusó a Descartes de blasfemia, crimen castigado por las leyes civiles del país, con la frase expresada en forma de tesis: eum esse blasphemum, qui Deum pro deceptore habet, ut male Cartesius ("Es blasfemo aquel que tiene a Dios por engañador, como el pésimo Descartes [ha dicho]"). Ante la gravedad de la situación 
En fin, estas reflexiones nos ayudan a comprender cómo la noción de un concepto sin objeto, nacido en el doble contexto del conceptualismo tardomedieval y de la teología voluntarista del siglo XIV, llegará en el siglo XVI hasta la filosofía de la segunda escolástica, y tras ella, a la filosofía moderna, propiciando aquel tipo de filosofía que ha reducido la entidad a objetividad del pensamiento y, consiguientemente, ha transformado la metafísica en teoría del conocimiento.

Leopoldo José Prieto López

Universidad Francisco de Vitoria

lprieto7@gmail.com

creada, la defensa de Descartes debió ser asumida por la embajada francesa en Holanda. Cf. R. Descartes, Carta a Elisabeth, 10 de mayo de 1647, AT, V, 15-19. 